\title{
Providing Presence Cues to Telephone Users
}

\author{
Allen E. Milewski and Thomas M. Smith \\ AT\&T Labs, Research \\ 100 Schulz Drive, Red Bank, NJ 07701 \\ (732) 345-3395 \\ allen@att.com tom@att.com
}

\begin{abstract}
A significant problem with telephone communication is that callers do not have enough awareness about the Personal Presence of people they want to call. The result can be unwanted, interrupting calls. The live addressbook is an application that helps users make more informed telephone calls and teleconferences, from anywhere, via their wireless PDA or desktop browser. Unlike other network-based address books, which maintain static information, the live addressbook can display dynamic information about where the recipient currently is (i.e., reach number), and how available he/she currently is for calls. The system accomplishes this by applying to telephony the "Buddy List" concepts made popular in Instant Messaging applications. User trials assess the applicability of Personal Presence information in a telephone context.
\end{abstract}

\section{Keywords}

Presence, Awareness, Telecommunication, Mobility

\section{INTRODUCTION}

It is generally agreed that communications between people is vitally dependent upon the context in which the communications take place [16]. Participants in face-toface conversations modify their behavior according to who is present, their partner's social mood and sense of urgency and many other things. Because of the importance of context in natural communications, there has been a significant amount of effort placed on understanding and adding context to technology-mediated communications. Most of this work has taken place in computer-supported collaboration systems $[6,9,12]$, and more recently in Chat and Instant Messaging systems. In this work, special attention has been directed at providing information about the status of others prior to communication. This has

Permission to make digital or hard copies of all or part of this work for personal or classroom use is granted without fee provided that copies are not made or distributed for profit or commercial advantage and that copies bear this notice and the full citation on the first page. To copy otherwise, or republish, to post on servers or to redistribute to lists, requires prior specific permission and/or a fee.

CSCW'00, December 2-6, 2000, Philadelphia, PA.

Copyright 2000 ACM 1-58113-222-0/00/0012 ..\$5.00. included information such as whether the person is in his/her office-typing on the keyboard, whether the person is already busy with another conversation, or whether the person is nearby a specific device. Just as physical and social cues facilitate the negotiation that begins face-toface conversations [5], this information might predict the likelihood that an invitation for technology-mediated communication will be accepted, and can even indicate how the conversation might proceed. The communication of this information has been termed "Personal Presence" or "Awareness."

In contrast to personal presence and awareness information explored by computer-mediated collaboration researchers, the telephone has very few such cues. Busy signals indicate one extreme form of unavailability, but generally, a telephone call has to be completed before its participants can begin negotiating it. Since telephone callers don't have enough information about the people that they want to call, they often make interrupting calls at unwanted times or to locations that their intended recipients have already left. The deluged recipients could react by turning their telephones "off," but they run the risk of missing important calls.

The live addressbook is a prototype telephone-manager designed to provide Personal Presence information to potential callers. Since the traditional telephone lacks the capability for setting or displaying personal presence, the live addressbook augments the telephone with a data device such as desktop browser or wireless PDA. As a research project, the live addressbook explores the usefulness of personal presence information in the realm of telephony and studies methods for letting users maintain their up-to-date presence information without undue overhead.

Designers of computer-based Personal Presence (Awareness) systems have traditionally been burdened with two major design tradeoffs. The first tradeoff, Informativeness vs Privacy, deals with the fact that if a person's status is conveyed fully enough to be useful to others, it may often violate that person's privacy. For example, when video is used as a highly informative, automatic means of reporting Personal Presence, it is 
common for users to report privacy concerns [11,14]. As a result, many systems have decreased the detail of Personal Presence information in order to increase privacy. This has been done by distorting the video [13], by using audio-only [1], and by reporting presence in a symbolic way or indirectly with avatars [4].

The second tradeoff, Overhead vs Control, has to do with how people maintain their own personal presence information. Obviously, a great deal of overhead is required to continually update one's status manually, as it changes throughout the day. Grudin [8] declared overhead to be one of the major drawbacks of most collaborative systems, and in order to eliminate this overhead, an assortment of techniques have been tried to provide automatic tracking of status. These include video [3], body movement-reporting [7], and active badges [10]. However, when personal presence information is recorded automatically, the user has less control over it. For example, there may be cases where the user would want to disguise his/her status from others. In this way, this tradeoff is related to privacy issues as well.

It is likely that providing Personal Presence information in a telephony setting has similar tradeoffs, although it is not clear that the same design solutions that apply to computerbased communication apply to telephone calls. For example, while Instant Messaging systems can rely on users logging in/off (or typing) as an important cue to presence, those cues are less applicable to telephony. Moreover, since the voice interaction associated with telephone calls often involve a more intense interactive experience than text-messages, failure to abide by, say, a "Do Not Disturb" signal may be more annoying in telephony than in text interactions. On the other hand, since telephone users have had a long history of usage without Personal Presence information, it is not clear that callers would utilize that information as much with the telephone as they do with computer-based communication. The live addressbook provides a useful environment in which to study these questions.

\section{SYSTEM DESCRIPTION}

A schematic of the live addressbook is shown in Figure 1.

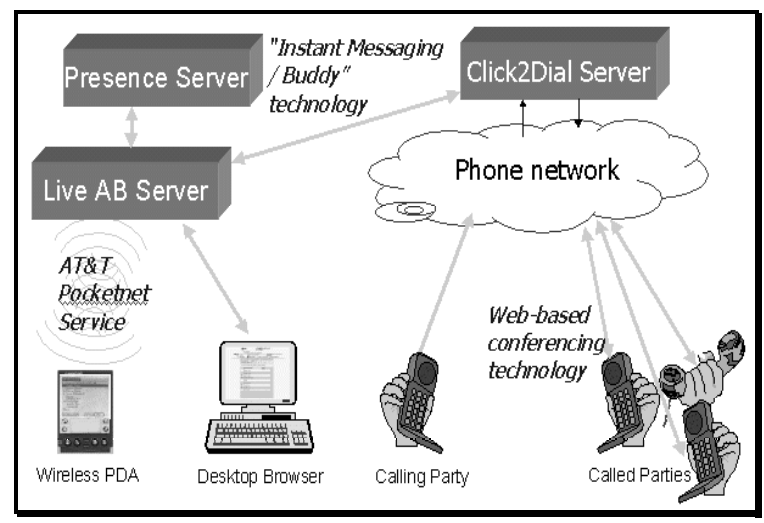

Fig. 1. Schematic of live addressbook
The system has several components:

\section{Network-Based Addressbook}

Users of the live addressbook can store information about contacts that they may want to call. These contacts fall into one of two categories. "Live contacts" are subscribers to the live addressbook. Live contacts are added to the addressbook simply by selecting their names from a list of all live addressbook subscribers. No other information must be entered about these contacts because the system maintains profiles for them. The system also supports "other contacts," a category that consists of any other (non-subscribing) people desired. For these contacts, users must enter their names and telephone numbers.

The contact information for each user is stored in network-based live addressbook server, and can be accessed from any web browser that connects to the server. Since the data is stored centrally, and not on individual devices, users can access exactly the same data from multiple devices. Moreover, they can update contact information from one device (e.g., office PC) and those updates are automatically available when accessing the addressbook from other devices (e.g., wireless PDA).

\section{Click-to-Dial Calling}

Unlike traditional addressbooks that provide you with a telephone number that, then, needs to be dialed on the telephone, calls are made directly from the live addressbook via "click-to-dial" technology. When a contact name is selected in the live addressbook, the live addressbook server send a signal to a telephony server/bridge which places two successive calls, one to the caller's current telephone and one to the callee's number. The calls are then bridged together. There are several advantages to using click-to-dial technology for this service. First, the PC or wireless PDA connected to the live addressbook can be used to control any telephone. This means that users might control their hotel-room telephone, or even a payphone at the airport with the same personalized visual user interface that they use in their office. Second, the use of click-to-dial calling enables certain calling capabilities that would not be possible in point-to-point calling; for example, conferencing calling.

\section{Conferencing-by-Default}

Bennett and Klinger [2] have suggested that a cognitively natural call model would include the concept that all calls are, by default, conference calls. This notion is implemented by the live addressbook using a multiparty Conferencing bridge as part of the Click-to-Dial server. After a user has called one person by selecting a name, additional people can be added simply by selecting more names. No special setup is required to distinguish a call to one versus multiple people, and people can be added or dropped at any point in the call. A "Current Call" 
screen shows the names of all people on the call, and the caller can drop individual people, or the entire call.

\section{Personal Presence Information}

Live addressbook users can set three entries as part of their personal presence information.

- Availability status refers to the user's ability and/or desire to accept telephone calls from others. We have limited Availability to four values: Available [+], Urgent Only [!], Away (leave message) [@] and Do Not Disturb [-]. When a user sets his/her availability status, it is displayed in the live addressbooks of other subscribers. In Figure 2, the icons to the left of entries represent the availability of this user's live contacts. Since other have no availability settings, there are no icons next to some names. An icon in the top portion of the screen indicates the user's own availability status. Availability is not technically enforced, but is meant as a cue for social interaction.

- Location refers to the telephone number at which a user can currently be reached. This number is used both as the number called first by the click-to-dial server when placing a call, and the one dialed when another subscriber tries to reach the user. That is, when a live contact is called, it is based on a dynamically current telephone number set by the live contact him/herself rather than on a static number entered by the caller. Locations can be given any name the user chooses (e.g. "home," "office," "mobile," etc.). In Figure 2, this user's current location is "office." The locations of other live contacts are displayed in popup windows (not shown) as the user rolls the mouse over live contact names.

- Message is a brief, free-form text message in which the user can type anything to augment the other personal presence information. Unlike Availability status and Location, the message is optional. In Figure 2, this user's message is set to "working." Messages set by other live contacts is shown in the pop-up window. Also shown in that window is the amount of time since Personal Presence information has been updated.

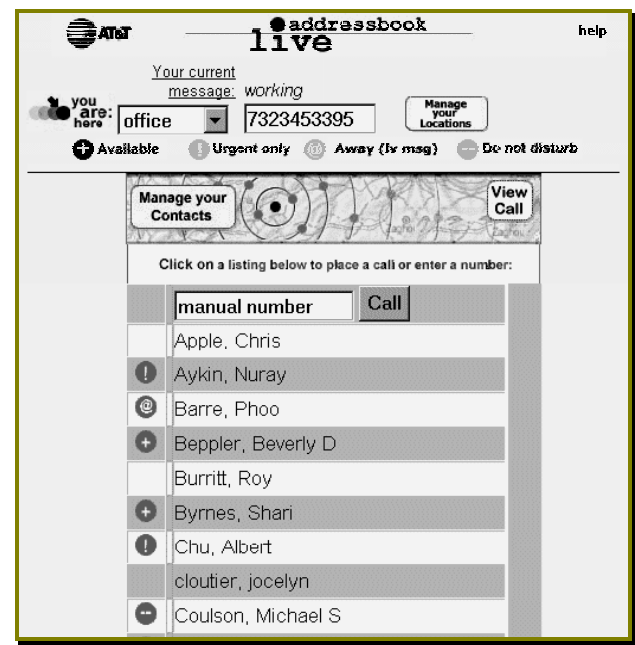

Figure 2. Web Browser Screen

\section{Overhead Reduction}

An essential factor in the utility of the live addressbook is that personal presence information be easily updated and kept current by live contacts. There are two ways in which the live addressbook attempts to keep the overhead of these updates low.

\section{Ubiquitous, Multiple-device Access}

We have simplified the updating of personal presence information by making it possible to do so from many different devices. Figure 2 shows the desktop webbrowser interface. The top portion of that screen can be used to change Availability status, Location or Message. The bottom portion lets the user telephone addressbook entries or manually entered numbers.

Another desktop PC tool is the live update-PC application, which sits in the Microsoft Windows "tray" at the bottom of the screen. Clicking on it reveals a menu of Availability status and Location choices. In addition, the user can change his/her Message from the same menu. An advantage of a "tray" application is that it is always running (executed at Startup) and therefore can often be more quickly used than the WWW browser. It is also more readily visible on a crowded PC screen. The tray icon displays the users current status symbolically, (e.g., +, !, @, -). 


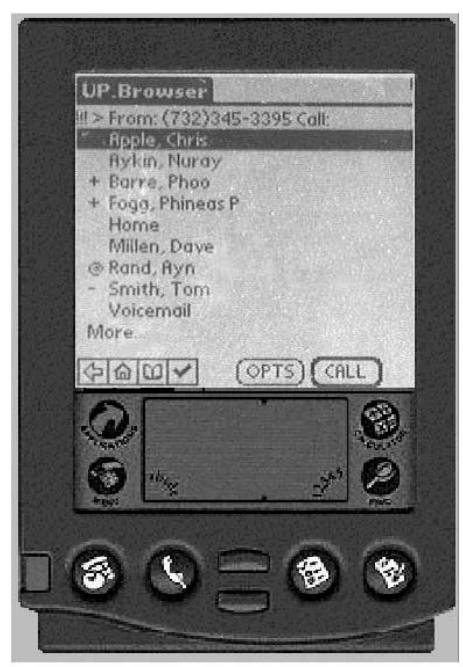

Figure 3. Palm Browser Screen

The live addressbook is also available from a wireless PDA. We designed the application for a Palm device with CDPD wireless modem. This device is quite portable and fits in one's pocket. Figure 3 shows the Palm screen while accessing the live addressbook. In addition to being able to make calls, the Palm version lets users update their Personal Presence information from anywhere. The live addressbook for the Palm relies on a web browser, as does the desktop Web version.

Finally, in addition to the PC and wireless PDA access, there is telephone access to the live addressbook. By calling an ' 800 ' number from any telephone, users can change personal presence information by selecting voice menu items. In addition, the presence information of live contacts can be accessed, and point-to-point calls can be made.

\section{Hint-Based Prompting}

In general, our strategy has been to avoid automatic means of updating personal presence information because it lessens the user's control and effects privacy. Instead, we have tried to make manual updating as simple as possible. We have included some semi-automatic detection of presence, but have used this information to prompt users to make changes, rather than automatically registering their Presence information.

The main prompting method is based on the fact that a live addressbook Location, in addition to being a telephone number, can also include the PC (or wireless PDA) that the user wants to associate with the telephone. For example, the user can associate his/her office PC with the office telephone number, or a wireless PDA with a cell phone. Once the user has configured this association, access from the PC or PDA can be used as a hint that the user might be at that Location. The live addressbook makes use of this scheme in three situations:
- When the user is currently registered as being at one location but accesses the live addressbook web page from a PC associated with a different location.

- When the user is currently registered as being at one location associated with a PC, but accesses the live addressbook from the Wireless PDA.

- When the user is registered as being at one Location, and then starts keyboard activity on a different PC with the live update-PC program running.

Each of these cases indicates a potential change in locations, and in each, the user is prompted to change their location. The prompt for the third (live update-PC) case is shown in Figure 4.

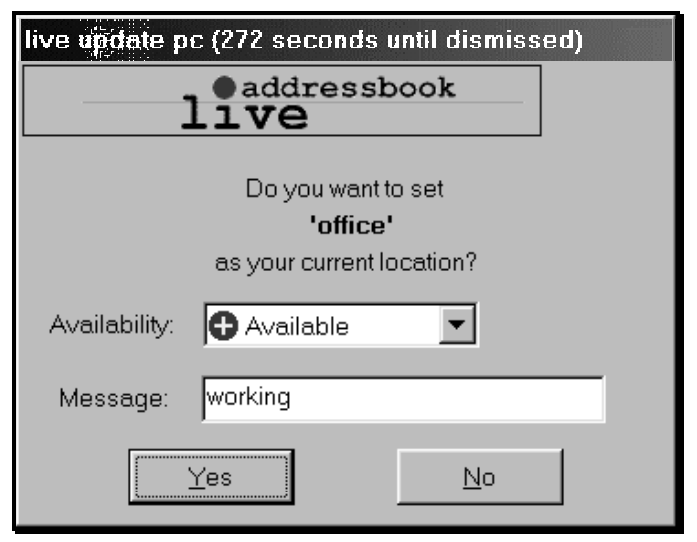

Figure 4. live update-PC prompt

\section{Evaluation}

The live addressbook was evaluated in a technical trial that lasted six weeks. A formal training session, held for approximately one-half of the participants, described the system's features and how to use them. The remaining users received informal training. Participants were asked to use the system as much as they found useful and, if possible, to make it a daily tool for work as well as for personal use. Throughout the trial, usage logs were automatically collected. These logs showed details on all calls made with the live addressbook and also recorded the date and time of all changes in Personal Presence. Finally, questionnaire and interview data were collected at the end of the six-week period. Twenty-two users were initially given the opportunity to use the live addressbook; we report only on the fifteen users that made at least one telephone call with it during the first two weeks of our trial period.

The trial concentrated on three groups. One workgroup ("Dispersed") included five members based in different locations as well as members who moved frequently between locations. Before including the live addressbook as a tool, this team held weekly, scheduled teleconferences and used email and Instant Messaging heavily in order to communicate. The second workgroup 
("Colocated") generally had colocated offices, though a few of the seven members traveled frequently. This group included a range of job functions and their collaboration was less self-contained than the "Dispersed" group. This group frequently used telephone calls and email, but seldom used Instant Messaging. Most of the members of this group had wireless PDAs; members of the other groups did not have wireless PDAs. The third group ("Misc") consisted of three people. Two of these people collaborated across coasts in a management-employee relationship. The third member was not part of a workgroup, but interacted with a variety of other live addressbook users.

\section{Using and Updating Presence Information}

The live addressbook project was conducted to explore the usefulness, for telephone calls, of the kind of presence information typically available in computer supported communication tools. Generally, our users reported that the system was useful and convenient. System usage varied widely: participants made between one and sixteen telephone calls per week with a mean of four calls per week. Users' reports and usage patterns provide a number of insights relevant to the design of Personal Presence systems.

1. Users will attempt to keep Personal Presence Information current, especially if the overhead of doing so is not large. Our users updated their Presence information a moderate but regular amount during the trial. The average participant made 1.4 updates per day, but individuals ranged from a mean of 4.3 updates per day down to two updates during the entire trial period. Figure 5 shows the relative frequency of reasons for updating Personal Presence information. By the far, most of the updates $(69 \%)$ were unprompted. The large proportion of unprompted updates indicates that our participants did make an active attempt to keep their presence information upto-date. On the other hand, prompting facilitated updating, since $31 \%$ of updates were prompted.

The most common way of updating one's Personal Presence was via a desktop PC (91\% of updates). Updates via the live update-pc application were the most common, followed closely by updates via the browser application. This might be expected since most participants spent most of their time at a desktop PC. Nonetheless, the number of updates made from the PDA and telephone interfaces was not negligible for those participants with appropriate equipment. Interviews suggested that these interfaces, while used much less frequently, were occasionally critical, especially when travelling between locations.

Figure 6 shows that Location updates were nominally more frequent than Availability updates. This difference was especially true for the "Dispersed" Group, whose members updated Location an average of 23.6 times, but who changed Availability only 12.8 times during the trial. It may be that Location changes are more critical, especially when mobile. In addition however, we speculate that Availability was kept less current than Location because it required more overhead to do so. First, Availability changes more frequently and less predictably than Location. Moreover, our "prompting" system was based solely on presumed changes in Location, as we found it difficult to estimate Availability automatically.
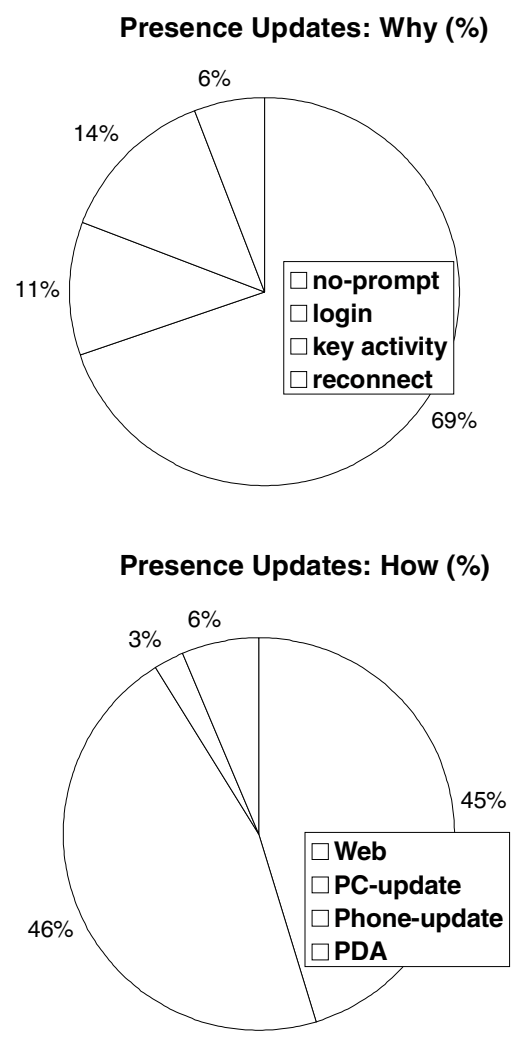

Figure 5.

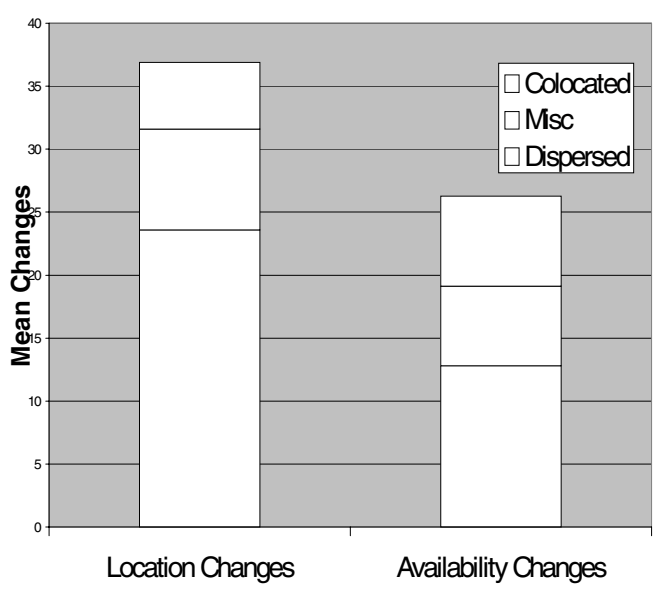

Figure 6. 
2. Both Personal Location and Availability Information are useful for telephone communications, but Availability is often too irregular to be reliable. Interviews and questionnaires indicated that the most useful and popular aspect of the live addressbook was the dynamic Location information maintained for live contacts. Interviews consistently revealed that participants liked not having to keep track of others' current location in order to telephone them. This was especially true for members of the mobile, noncolocated "Dispersed" Group. Several participants cited the lack of Location information as a shortcoming of traditional telephony.

The usefulness of the live addressbooks's Availability information is much less clear. Nominally, there were fewer calls made to participants set to "Do Not Disturb" and "Away" than to those set to "Available" and "Urgent." Users also spent less time in the "Do Not Disturb" and "Away" states. Normalized for differences in the amount of time spent in each state, an average of $72 \%$ of calls were received while in the "Available" or "Urgent" states. However, large individual differences prevent this difference from reaching significance. Participants reported liking the Availability information, but not using it much because it was "unreliable." Only a few (mostly in the "Colocated" Group) reported checking Availability information before making calls. Most users claim they did not. A common complaint was that Availability information was not worth checking because it was not kept up-do-date by others. One participant described a common scenario in which she attempted to reach a colleague, who was set to "Do Not Disturb," but the timestamp indicated that the last update was several days prior. She decided to call anyway, and found him available.

3. Interactive negotiation of Availability may be superior to Availability updates/displays. One of our user groups (the "Dispersed" Group) developed a fascinating strategy. Since they were already heavy users of Instant Messaging (IM) for inter-group communications, they found that much more credible Availability information could be gathered from that tool. Specifically, they would send an IM message to probe the availability of the potential recipient (e.g., "can I call you?"). If they received a positive response to the IM, they would then use the live addressbook to place the telephone call, relying upon its Location information. This is a particularly interesting example of the "outeraction" reported by Nardi, Whittaker, and Bradner elsewhere in these proceedings [15]. Here, users had an alternative means of checking Presence that is, seemingly, easier than IM. Apparently, members of this workgroup considered the task of responding to the "probe" IM to have low overhead, and they noted in interviews, that the interactivity of IM gives it unquestionable credibility. Moreover, they felt that the real-time, on-demand nature of this response lets potential recipients assess their own availability at the instant of the call. This, they felt, better matched the rapidly changing nature of availability more conveniently than attempting to keep their displayed Availability up-to-date.

It is possible that the rapidly-changing nature of Availability does not lend itself to the "update and display" type of interface provided by the live addressbook (and many IM systems as well). There is potential for either integrating interactive text messaging as a means of negotiating calls, or for providing audio screening.

4. Mixing automatic-detection and manual updating capabilities is a useful overhead-reduction strategy. While many presence updates occured unprompted, approximately thirty percent of them resulted from prompting by the live addressbook system. One-half of these occurred while participants accessed either the desktop browser application, the live update-pc, the telephone or PDA application. The other half occurred as a result of participants' keyboard activity at a desktop PC other than the one they were currently registered at. Interviews indicated that prompting was a useful reminder to update presence. Moreover, participants did not complain that the prompting was intrusive. Indeed, the majority of participants interviewed preferred our strategy of prompting simple manual updates to a potential automatic-update strategy. For example, one participant understood that the live addressbook could misdirect calls if presence information was set incorrectly, which she considered a serious social error. She felt the error would be even more serious if caused by "a computer," and preferred the personal control that prompting provides. The prompting we did required minimal overhead for users to respond and helped them remember to update Presence at critical times. Automatic updating without prompting was considered too intrusive and potentially dangerous.

\section{Other Findings}

1. Telephone "buddy lists" should not be separated from other telephoning interfaces. People are used to being able to call anyone from a telephone. Our users made calls, not just to "live contacts," but to "other contacts" and manually-entered numbers as well. They reported liking the ability to make all these calls from a single interface, Therefore, while a "buddy-list" subset of close telephone contacts is useful, it needs to integrated into a larger, more universal communication system. 
2. Support for communication and presence-updating capabilities from multiple devices is useful. While the desk telephone and desktop browser were the most common method of calling and updating, our users also found great value in the telephone and PDA interfaces. These interfaces provided consistency, even when travelling away from the desktop. Moreover, they made it much easier to keep Personal Presence information up-to-date.

3. Telephone calls and Instant Messages have different dynamics. Our trial was suggestive of several ways in which telephony and Instant Messaging may differ, even though Presence information is useful for both. First, of course, is the fact that IM systems have been able to utilize user logins as an indication of presence, while telephony has no such analog. In addition, there was a preference of our users to include both "live contacts" and other addressbook information all in one interface. This differs from IM systems, which are largely closed systems that do not provide universal access. Finally was the "Dispersed" Group's tendency to use IMs to negotiate telephone calls. Apparently, an IM was considered less intrusive (and possibly easier) than simply telephoning the recipient directly. This practice may point to differences inherent in the text and interactive-audio media. Further study is required to clarify these differences.

4. Multiparty communications support is important for workgroups. Our users found the conference-calling feature to be very useful for group collaboration. However, we did not find strong workstyle changes resulting from the ability to spontaneously turn any telephone call into a conference call. While there was some spontaneous conferencing, most conferences were planned, or even prescheduled.

5. Work styles differ between groups; it is critical to design a flexible service to support these differences. Perhaps the most consistent finding across our trial was that individuals and groups utilized telephony and Personal Presence information differently, depending on their needs and contexts. How groups used the system differed depending on how geographically dispersed members were and how experienced members were with Instant Messaging technologies. More importantly, while all users reported liking the live addressbook, they reported liking it for different reasons: Location information, click-to-dial convenience, conferencing, PDA-access, etc. This points to the necessity to provide a flexible set of capabilities that can be modified to fit the work context of the specific group.

There is great potential for presence-enabled tools as a way of simplifying our communications and making them more efficient. Building useful tools requires a good understanding of how people use Presence information in the context of different communication media. The live addressbook has begun exploration of this usage in a telephone-only context. Further study is needed to understand the complex interactions of presence, media and differences in work style.

\section{References}

1. Ackerman, M. S., Hindus, D., Mainwaring, S. D., \& Starr, B. Hanging on the Wire: A Field Study of an Audio-Only Media Space. ACM Transactions on Computer-Human Interaction, vol. 4 (1997), 39-66.

2. Bennett, R.W. and Klinger, J.G. Telephony Interfaces derived from user's conceptual models. Proceedings of the AT\&T Behavioral Sciences Days (1990), 45-49.

3. Bly, S. A., Harrison, S. R., \& Irwin, S. Media Spaces: Bringing People Together in a Video, Audio, and Computing Environment. Communications of the ACM, 35(1), 1993. 28-46.

4. Broll, W., Grather, W., Meir, E., Pankoke-Babatz, U. and Prinz, W. Place people in context-the awareness forum. Proceedings of Meetings of the Human-Computer Interface International (1999), 462-471.

5. Clark, H. Using Language. Cambridge University Press, 1996.

6. Erickson, T. Smith, D.N., Kellogg, W.A., Laff, M., Richards, J.T. and Bradner, E. Socially Translucent Systems: Social Proxies, Persistent Conversation and the Design of "Babble." Proceedings of the CHI99 Conference on Human Factors in Computing Systems, ACM Press (1999), 72-79.

7. Greenberg, S. Peepholes: Low Cost Awareness of One's Community. Conference Companion of the CHI '91 Conference on Human Factors in Computing Systems, ACM Press (1991), 206-207.

8. Grudin, J.,. Groupware and Social Dynamics: eight challenges for developers. Communications of the ACM (1994), 92-105.

9. Gutwin, C. \& Greenberg, S. Workspace Awareness for Groupware. Proceedings of the CHI '96 Conference on Human Factors in Computing Systems,. ACM Press (1996), 208-209.

10. Hopper, A., Harter, A., and Blackie, T. The active badge system, Proceedings of CHI '93. Conference on Human Factors in Computing Systems (1993), 533-534

11. Hudson, S. E., and Smith, I. Techniques for Addressing Fundamental Privacy and Disruption Tradeoffs in Awareness Support Systems. 
Proceedings of Computer Supported Cooperative Work '96, ACM Press (1996), 248-257.

12. Karsenty, A. Easing interaction through groupawareness. Proceedings of the International User Interaction (1997), 225-228.

13. Lee, A., Girgensohn, A. \& Schlueter, K. NYNEX Portholes: Initial User Reactions and Redesign Implications. Group'97 Proceedings of the International ACM SIGGROUP Conference on Supporting Group Work, ACM Press (1997), 385-393.

14. Millen, D., Milewski, A., Smith, T., Weimer, D., Wellner, P. The Corner Office: An Exploration of an
Informal Teleconferencing Service. Proceedings of 17th International Symposium on Human Factors in Telecommunications, Copenhagen, Denmark, May 4-7, 1999, 103-112.

15. Nardi, B., Whittaker, S. \& Bradner, E. Interaction and Outeraction: Instant Messaging in Action. Proceedings of Computer Supported Cooperative Work 2000, ACM Press (2000).

16. Suchman, L. Plans and Situated Actions: The problem of human-machine communication. Cambridge University Press, 1987. 